\title{
Diagnosing lleal Atresia: The Role of Clinical Correlation and Plain Radiography
}

Timothy Mwai Jumbi ${ }^{1}$, Manvinder Singh Mann ${ }^{1}$, Joel Kipyegon Lessan ${ }^{2}$

1. University of Nairobi

2. Kenyatta National Hospital

Correspondence to: Dr Timothy Jumbi, University of Nairobi P.O Box 1059- 00618 Nairobi, Kenya. Email: mwaitim@gmail.com

\section{Summary}

Ileal atresia is a subset of congenital gastrointestinal malformations that result in neonatal bowel obstruction. We report a case of a 7-day old male neonate who presented with failure to pass meconium, abdominal distension and late onset bilious vomiting. A plain abdominal radiograph revealed dilated bowel loops with fluid levels of different sizes, the largest appearing as a fluid filled mass with an abrupt cut off. These findings were consistent with ileal atresia and the diagnosis was confirmed intra-operatively. This case description is aimed at emphasizing the role of clinical correlation and a plain abdominal radiograph in the diagnosis of ileal atresia which are two important modalities that have imaging modalities which delay management. The diagnosis of ileal atresia can be suspected in the neonate who presents with failure to pass meconium, abdominal distension, late onset bilious vomiting and a plain abdominal radiograph with fluid levels of different sizes, the largest situated just proximal to the atretic zone.

Key words: Ileal atresia, plain radiograph, clinical assessment

Ann Afr Surg. 2018;15(2):73-76

DOI:http://dx.doi.org/10.4314/aas.v15i2.9

(C) 2018 Author. This work is licensed under the

Creative Commons Attribution 4.0 International License.

Conflicts of Interest: None

Funding: None

\section{Introduction}

Small intestinal atresia is a congenital anomaly characterized by the abnormal closure, discontinuity or narrowing of the duodenum, jejunum or ileum. (1) The diagnosis of Intestinal atresia is based on clinical correlation and a plain radiograph. The value of these two modalities has been blurred by the irrational use of ultrasound and other imaging modalities. (2) More importantly the clinical signs and symptoms may provide a clue as to the site of intestinal atresia while the radiograph confirms the diagnosis. High intestinal atresia include duodenal and jejunal atresia while low intestinal atresia includes distal ileum and colonic atresia. (3) We review the importance of a plain radiograph and clinical assessment in a neonate with ileal atresia with an aim of emphasizing that unnecessary imaging investigations do not add value in a clear-cut diagnosis.

\section{Case Presentation}

A 7-day old male infant was referred to the newborn unit of Kenyatta National Hospital, Nairobi Kenya. The reason for referral was failure to pass meconium since birth requiring further investigation and management. The child also had progressive abdominal distension over the 6 days and associated vomiting that was initially non-bilious but progressed to become bilious on the 5th day of life. Details of any relevant pre-natal occurrences and events during delivery or immediately after birth are unclear as the infant was born at home and the mother did not attend any antenatal care clinic. On examination the neonate was stable, well hydrated with warm extremities. The abdominal examination revealed a grossly distended abdomen with visible bowel loops but otherwise non tender (figure 1). The bowel sounds were present but reduced and a rectal 


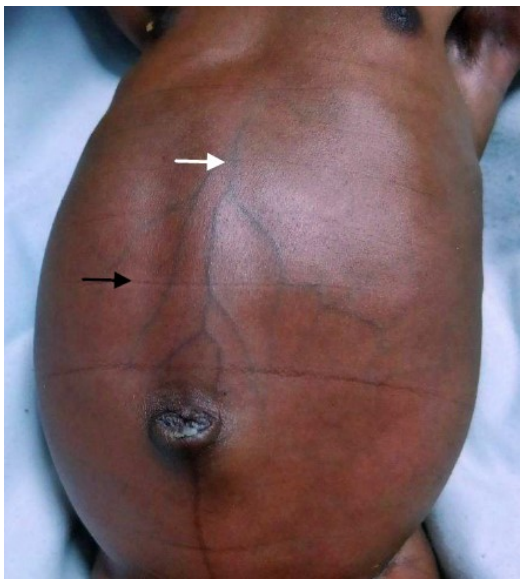

Figure 1: Abdomen at initial presentation with visible bowel loops (black arrow) and visible veins (white arrow)

exam revealed a normally placed anus of normal tone, an empty rectum with no flatus or stool on withdrawal of finger. There were no other anomalies noted on systemic enquiry or examination. Notably, the child presented to us with both a plain abdominal radiograph and an abdominal ultrasound done in a peripheral facility prior to referral. The ultrasound was inconclusive but the radiograph displayed dilated bowel loops, increased bowel wall enhancement and a significant fluid filled dilated loop approximately $25 \mathrm{~mm}$ in diameter with an abrupt cut-off. No gas or stool was noted in the colon and rectum (Figure 2). The findings on clinical assessment and imaging were consistent with ileal atresia and the patient was

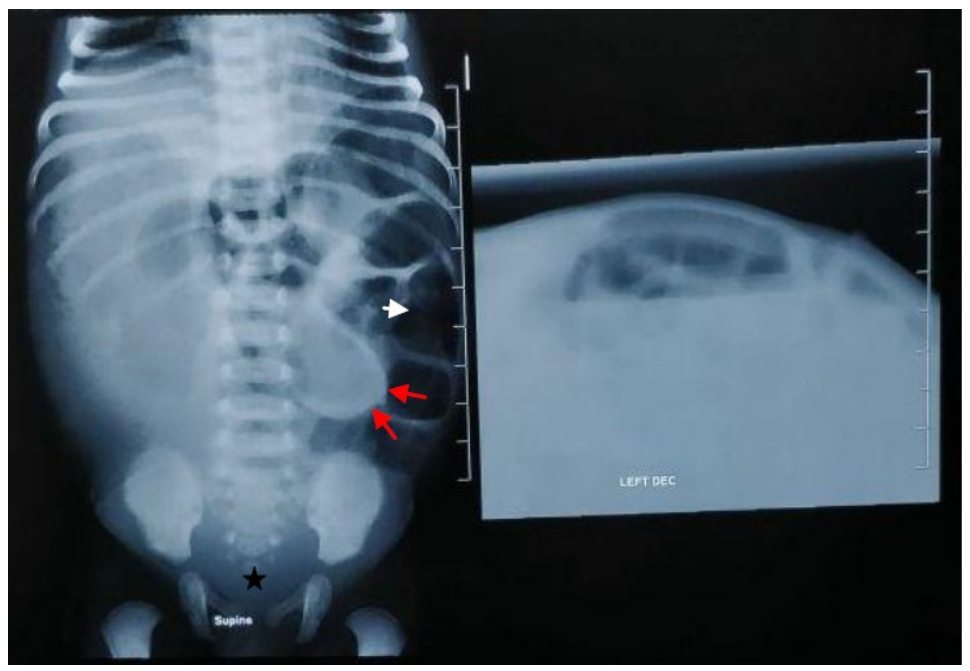

Figure 2: supine and lateral Plain radiographs of the neonate demonstrate dilated bowel loops (white arrows) with a significant fluid filled dilated loop with an abrupt cut-off (Red arrows) There is absence of distal gas in the colon and rectum ( asterix) scheduled for surgery. After obtaining informed consent from the guardian, the infant had an exploratory laparotomy. Intra- operatively, we found a type $3 \mathrm{~A}$ ileal atresia at the terminal ileum with a $\mathrm{V}$ shaped mesenteric defect between the proximal and distal segment (figure 3). The atretic zone was $2 \mathrm{~cm}$ from the ileo-cecal valve. The proximal segment was dilated, filled with meconium with a blind end as correlated with the plain radiograph. The distal segment of ileum and colon was collapsed but confirmed to be patent till the rectum. Approximately $10 \mathrm{~cm}$ of the dilated proximal segment and $2 \mathrm{~cm}$ of the distal atretic segment was resected with the appendix and an end to side ileocecal anastomosis done. We initiated trophic feeds 72 hours after surgery which were well tolerated and by the 6th day the neonate had graduated to full oral feeds. We discharged on the 7th Post-operative day after a full recovery.

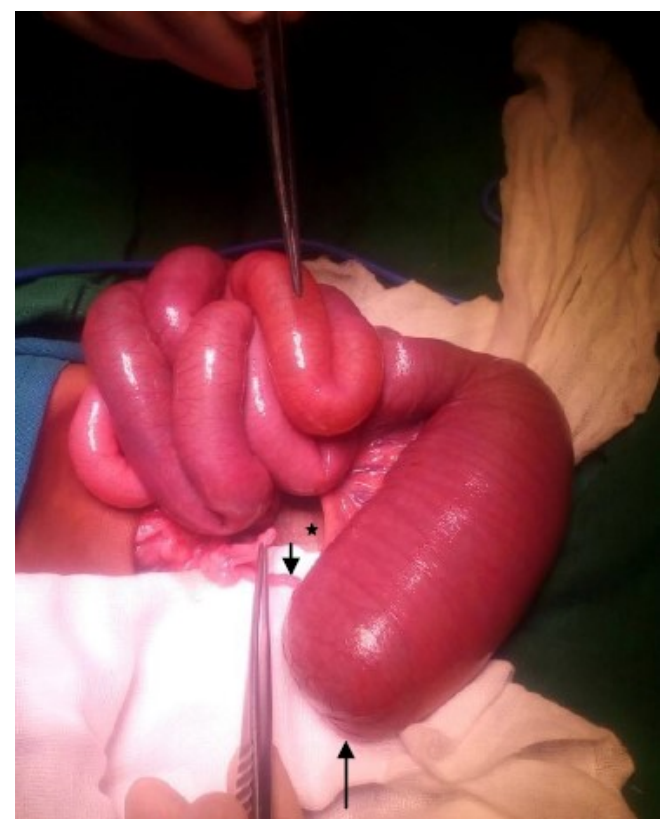

Figure 3: Intraoperative findings Type 3a ileal atresia with a Vshaped mesenteric defect (asterix). The proximal segment is dilated filled with meconium (long black arrow) the collapsed distal segment is held with a forceps and is $2 \mathrm{~cm}$ from the ilealcecal valve. The appendix can be visualized (small black arrow).

\section{Discussion}

Jejuno-ileal atresia is a subset of congenital gastrointestinal malformations that result in neonatal bowel obstruction and occurs with an incidence of 0.7 
per 10,000 births. (4) Isolated jejuno-ileal atresia is known to occur as a result of intra-uterine mesenteric vascular accidents. (5) The current classification is described by Grosfield and colleagues as follows; Type I is a mucosal atresia with intact bowel wall and mesentery. Type II has two blind ends which are separated by a fibrous cord. Type IIIa has two blind ends which are separated by a V-shaped mesenteric defect. Type IIIb is an apple-peel atresia and Type IV represents multiple atresias.(7)

The post-natal diagnosis of ileal atresia is made by clinical signs and symptoms correlated by imaging findings. Majority of infants with ileal atresia fail to pass meconium in the first 24 hours of life, in some instances either meconium or necrotic tissue is occasionally passed. (6) Abdominal distention that occurs with ileal atresia is global and generalized as opposed to distension limited to the upper aspects of the abdomen or otherwise absent which is often associated with a more proximal atresia. The abdominal distension usually precedes the onset of vomiting and may be associated with visible bowel loops and peristalsis. (7) A review done by Lucinda and Stanton reports that distal ileal or colonic atresia present with late onset bilious vomiting evident past day 4 of life. (8) These features were consistent with findings in our patient who presented with failure to pass meconium, gross abdominal distension progressively increasing in size and late onset bilious vomiting. An initial plain radiograph can be entirely diagnostic with no further evaluation required. Supine and lateral decubitus abdominal radiographs are obtained in each case with the clinician paying attention to the gas and fluid patterns displayed. (9)Ileal atresia would result in multiple, asymmetric centrally placed fluid levels that are often of different sizes. The largest fluid level is just proximal to the site of obstruction which is an important sign on a radiograph that can be seen as fluid filled structure with an abrupt distal cut off. Additionally the absence of colonic or rectal gas demonstrates a complete obstruction located at the small intestine. (2) The radiograph in our case displayed these important signs that were key in confirming the diagnosis. Other imaging modalities have limited to no role in the diagnosis of ileal atresia and often delay management in such patients. Ultrasonography in the postnatal period is of limited value particularly in distal obstruction due to introduction of air to the gut which absorbs the ultrasound beam.(10) The exception is prenatal ultrasonography which has been shown to have a role in the diagnosis of jejuniileal atresia.(11) Contrast studies are also not indicated unless in non specific conditions of low intestinal obstructions in which differentiating between dilated loops of small and large intestine is difficult. (12) There was no indication for such contrast studies in our patient as it was clear from the clinical presentation and plain radiograph that there was complete intestinal obstruction. There is no role of CTscan and MRI in intestinal atresia but they can provide additional anatomic detail in other congenital anomalies of the gastrointestinal tract such as malrotation and anorectal anomalies.(13)

\section{Conclusion}

The diagnosis of ileal atresia is based on clinical assessment correlated with imaging findings and can be suspected in the neonate who presents with failure to pass meconium, abdominal distension, late onset bilious vomiting and a plain abdominal radiograph with fluid levels of different sizes, the largest situated just proximal to the atretic zone.

\section{References}

1. Milind K. Duodenal and Small Intestinal Atresia. Surgery (Oxford). 2010;28:33 - 37

2. Prasad GR, Aziz A. Abdominal Plain Radiograph in Neonatal Intestinal Obstruction. J Neonat Surg. 2017; 6:6.

3. Vecchia LK, Grosfeld JL, West KW, Et Al Intestinal Atresia and Stenosis. A 25-Year Experience With 277 Cases. Arch Surg. 1998; 133(5):490-97.

4. Best KE, Tennant PG, Addor MC, Et Al. Epidemiology of Small Intestinal Atresia in Europe: A Register-Based Study. Arch Dis Child Fetal Neonatal Ed.2011-300631

5. Louw J. H,Barnard $\mathrm{CN}$. Congenital Intestinal Atresia; Observations on its Origin. Lancet 1955; 269(6899)1065-7 
6. Baucke V, Kimura K. Failure To Pass Meconium: Diagnosing Neonatal Intestinal Obstruction. Am Fam Physician. 1999;60(7):2043-50.

7. Frischer JS, Azizkhan RG. Jejunoileal Atresia and Stenosis. In: Coran AG, Adzick N S, Eds. Pediatric Surgery. Philadelphia, PA: Elsevier Mosby;2012.Pp1059-71

8. Lucinda G.C, Stanton MP. Bilious Vomiting in The Newborn. Surgery (Oxford).2016; 34(12): 603-8

9. Harlow CL, Stears RL, Zeligman BE, Archer PG. Diagnosis of Bowel Obstruction on Plain Abdominal Radiographs: Significance Of AirFluid Levels At Different Heights In The Same Loop Of Bowel. AJR Am J Roentgenol. 1993; 161:2915.

10. Neal MR, Seibert JJ, Vanderzalm T, et al. Neonatal Ultrasonography to Distinguish Between Meconium Ileus and Ileal Atresia. J Ultrasound Med. 1997;4:263-6.

11. Virgone C, D'Antonio F, KalhilAet al. Accuracy of Prenatal Ultrasound in Detecting Jejunal and Ileal Atresia: A Systematic Review And Meta-Analysis. Ultrasound Obstet Gynecol. 2014;45(5):523-9.

12. Bernard F. Laya, D.O., Mariaem M. Andres, M.D., Et Al. Patterns of Microcolon: Imaging Strategies For Diagnosis of Lower Intestinal Obstructioniln Neonates. J Am Osteopath CollRadiol 2015; 4(1):1-11

13. Berrocal T, Lamas $M$, Gutiérrez $J$ Et Al. Congenital Anomalies of The Small Intestine, Colon, and Rectum. Radiographics 1999;19(5): 1219-36 